\title{
ARTICLE \\ Motivational changes that develop in a mouse model of inflammation-induced depression are independent of indoleamine 2,3 dioxygenase
}

\author{
Elisabeth G. Vichaya (D) ${ }^{1}$, Geoffroy Laumet ${ }^{1}{ }^{1}$, Diana L. Christian¹, Aaron J. Grossberg ${ }^{1}$, Darlene J. Estrada ${ }^{1}$, Cobi J. Heijnen ${ }^{1}$, \\ Annemieke Kavelaars ${ }^{1}$ and Robert Dantzer ${ }^{1}$
}

Despite years of research, our understanding of the mechanisms by which inflammation induces depression is still limited. As clinical data points to a strong association between depression and motivational alterations, we sought to (1) characterize the motivational changes that are associated with inflammation in mice, and (2) determine if they depend on inflammation-induced activation of indoleamine 2,3 dioxygenase-1 (IDO1). Lipopolysaccharide (LPS)-treated or spared nerve injured (SNI) wild type (WT) and $I d o 1^{-/-}$mice underwent behavioral tests of antidepressant activity (e.g., forced swim test) and motivated behavior, including assessment of (1) reward expectancy using a food-related anticipatory activity task, (2) willingness to work for reward using a progressive ratio schedule of food reinforcement, (3) effort allocation using a concurrent choice task, and (4) ability to associate environmental cues with reward using conditioned place preference. LPS- and SNI-induced deficits in behavioral tests of antidepressant activity in WT but not $I d o 1^{-1-}$ mice. Further, LPS decreased food related-anticipatory activity, reduced performance in the progressive ratio task, and shifted effort toward the preferred reward in the concurrent choice task. These effects were observed in both WT and $I d o 1^{-/-}$mice. Finally, SNI mice developed a conditioned place preference based on relief from pain in an IDO1-independent manner. These findings demonstrate that the motivational effects of inflammation do not require IDO1. Further, they indicate that the motivational component of inflammation-induced depression is mechanistically distinct from that measured by behavioral tests of antidepressant activity.

Neuropsychopharmacology (2019) 44:364-371; https://doi.org/10.1038/s41386-018-0075-z

\section{INTRODUCTION}

Major depressive disorder affects $\sim 15.7$ million adults in the United States annually [15]. While the pathophysiology of this disorder is still unclear, chronic low-grade inflammation underlies the development of symptoms in a subset of individuals [1]. This is supported by increased prevalence rates for depression among individuals with inflammatory diseases and the ability of cytokine antagonists to alleviate symptoms of depression in these individuals independent of effecting the disease process [2]. Despite years of clinical and preclinical studies, our understanding of the relationship between inflammation and depression is limited. Population studies on the association of inflammation with specific symptoms of depression reveal that somatic symptoms (e.g., fatigue) show a stronger association with inflammatory markers than do affective symptoms (e.g., sadness) [3-5]. Further, somatic symptoms of depression, such as sleep and fatigue, tend to be more resistant to antidepressant treatment $[6,7]$. Further, inflammation in depressed patients is associated with decreased connectivity within corticostriatal circuits involved in reward and motivation [8]. However, little is known about the motivational aspects of inflammation-induced depression. The present study was undertaken to fill this knowledge gap. We focused on testing the effect of inflammation on components of positive valence systems as defined by the Research Domain
Criteria (RDoC) of the National Institute of Mental Health [9]. These systems regulate responses to positive motivational situations/ contexts and are disrupted in a variety of mental health disorders, including major depressive disorder.

Indoleamine 2,3 dioxygenase-1 (IDO1), which mediates the conversion of tryptophan to kynurenine, is required for the induction of inflammation-induced depression-like behavior as measured by behavioral tests of antidepressant activity [10-12]. However, the contribution of this pathway to inflammationinduced motivational changes has not yet been addressed. Kynurenine within the brain is metabolized into neurotoxic metabolites (e.g., quniolinic acid) that act as $N$-methyl-D-aspartate (NMDA) receptor agonists to induce behavioral changes in these tests [13]. The role of this pathway in depression is supported by clinical data demonstrating increased kynurenine levels in cerebrospinal fluid of depressed patients [14] and increased neurotoxic kynurenine metabolites levels within brains of individuals who committed suicide [15]. We investigated the role of IDO1 in inflammation-induced motivational deficits by comparing $I d o 1^{-/-}$to wild type (WT) mice in behavioral tests measuring different components of the RDoC positive valence systems including: (1) reward expectance measured by a food-related anticipatory activity task; (2) willingness to work for reward assessed by a progressive ratio task; (3) reward valuation and

${ }^{1}$ Division of Internal Medicine, Department of Symptom Research, The University of Texas MD Anderson Cancer Center, Houston, TX 77030, USA

Correspondence: Elisabeth G. Vichaya (egvichaya@mdanderson.org)

Received: 2 January 2018 Revised: 18 March 2018 Accepted: 16 April 2018

Published online: 27 April 2018 
effort allocation evaluated by an effort-based decision making task; (4) ability to associate environmental cues to reward using a conditioned place preference (CPP) paradigm. Inflammation was induced by systemic administration of lipopolysaccharide (LPS) or spared nerve injury (SNI). These treatments induce depression-like behavior, as measured by behavioral tests of antidepressant activity, via an IDO1-dependent mechanism [11-13]. Our results demonstrate that inflammation alters reward expectancy, willingness to expand effort for reward, and effort allocation without compromising reward learning and that these effects are independent of IDO1.

\section{METHODS AND MATERIALS}

Experimental subjects

Experiments used male and female adult WT C57BL6/J mice and $1 d o 1^{-1-}$ mice bred in-house, originally purchased from Jackson Laboratory. Mice were maintained on a 12-h light:dark cycle. Male mice were used in studies of LPS treatment and female mice in studies of SNI. Our SNI findings parallel and extend those previously reported in male mice [12]. Besides a possible decreased activity of the neuroprotective branch of the kynurenine pathway in females, potentially making them more susceptible to IDO-dependent inflammation-induced depression [16], we did not expect an interaction between sex and inflammation-induced activation of the kynurenine pathway.

Models of acute and chronic inflammation

LPS-induced model of acute inflammation: single-housed male mice were treated with LPS (serotype 0127:B8; Sigma-Aldrich, St. Louis, MO) or PBS by intraperitoneal (IP) injection. At the time of testing mice were 14-18 weeks of age. A $0.33 \mathrm{mg} / \mathrm{kg}$ LPS dose was selected as it induces liver Ido1 (Figure S1), induces brain inflammatory cytokines [17, 18], and causes depression-like behavior in behavioral tests of antidepressant activity [17-19]. Mice were tested around $24 \mathrm{~h}$ after treatment when these tasks have previously been shown to be IDO1dependent [20].

SNI model of chronic inflammation: we have previously shown that SNI-induces IDO1-dependent depressive-like behavior in behavioral tests of antidepressant activity [12]. SNI was performed as previously described $[12,21]$. Briefly, 10 -week-old female mice were deeply anesthetized by isoflurane and the common peroneal and tibial nerves were ligated and then transected, leaving the sural nerve intact. Sham mice underwent surgery without nerve ligation/transection. Mice were group housed (3-5/cage) for 4 weeks post-surgery and then single-housed for testing 5-6 weeks post-surgery.

\section{Behavioral testing}

Behavioral tests of antidepressant activity: behavioral tests developed to screen for antidepressant activity of psychotropic drugs (e.g., forced swim test (FST), sucrose preference, and novelty suppressed feeding (NSF)) were used to confirm the role of IDO1 in inflammation-induced depression. We refer to them as behavioral tests of antidepressant activity even if they are often used for testing for depression in rodents.

For FST, each mouse was placed in a swim chamber filled with $24 \pm 1{ }^{\circ} \mathrm{C}$ water. Immobility time during the last $5 \mathrm{~min}$ of a 6 -min test was scored as previously described [11, 13]. FST was conducted $24 \mathrm{~h}$ after $0.33 \mathrm{mg} / \mathrm{kg}$ LPS and 6 weeks after SNI.

For the sucrose preference test, mice were provided with a water bottle and another bottle with $1 \%$ sucrose solution. Consumption was measured in $12 \mathrm{~h}$ intervals after treatment with $0.33 \mathrm{mg} / \mathrm{kg}$ LPS $[13,22]$.

The NSF task was conducted 6 weeks after SNI surgery $[23,57]$. Mice were food deprived for $24 \mathrm{~h}$ and then placed in a novel cage with fresh bedding $(50 \times 30 \times 30 \mathrm{~cm})$. A food pellet was placed in the center on a white paper square. Latency to start eating was recorded.

Locomotor activity: this activity was assessed for $5 \mathrm{~min}$ in an empty arena $(18.4 \times 29.2 \mathrm{~cm}$ for LPS; $50 \times 30 \mathrm{~cm}$ for SNI). Activity was recorded by video camera, and distance traveled was quantified using Noldus EthoVision XT Software (Nodus Information Technology, Leesberg, VA).

Assessment of evoked and spontaneous pain: pain was assessed 5 weeks after SNI. Evoked pain was assessed by von Frey test as previously described $[21,24,25]$. Briefly, mice were acclimated to chambers on a raised mesh floor and were presented a series of calibrated von Frey filaments to the plantar surface of the hind paw. A brisk paw withdrawal or flinch constituted a positive response. In the absence of a response a filament of greater force was applied. In the presence of a lower force filament was applied. The $50 \%$ withdrawal threshold was determined using the updown method.

Non-evoked pain was assessed by using dynamic weight bearing system (BioSeb, Paris, France). Mice explored the enclosure for $10 \mathrm{~min}$. Floor-instrumented pressure data were collected during the last $5 \mathrm{~min}$ of exploration. Raw pressure data were automatically synchronized with images from the video camera and the averaged pressure values were computed by BioSeb software v1.3. Data are presented as percent body weight on the ipsilateral hind limb $[26,27]$.

Anticipatory activity task. Conditioned expectation of food delivery was induced by restricting food access to $4 \mathrm{~h}$ during the light cycle (zeitgeber time (ZT) 5-9) [28-30]. This schedule maintained mice at $85-90 \%$ body weight. Food-related anticipatory activity was quantified by the amount of locomotor activity in 5 min immediately prior to food delivery. After 2 weeks of restricted food access, mice were treated with LPS or PBS at ZT 7 and food was removed. Mice were tested the next day at ZT 5, when food was previously provided. For this experiment a $1.0 \mathrm{mg} /$ $\mathrm{kg}$ LPS dose was required as the $0.33 \mathrm{mg} / \mathrm{kg}$ dose was insufficient to reduce anticipatory activity in the context of combined food restriction and wheel running (Figure S2).

Progressive ratio task. The progressive ratio task was used to evaluate effort valuation. Mice were food restricted to $85-90 \%$ of free feeding weight and were trained to nose poke for chocolateflavored Dustless Precision Pellets $(20 \mathrm{mg}$, BioServ, Frenchtown, $\mathrm{NJ}$ ) in operant conditioning chambers equipped with a single nose-poke response unit and food dispenser (Med Associates, St. Albans, VT). Mice were trained on a fixed ratio 1 (FR1, 1 reward for 1 nose poke) schedule for 6 days, FR5 (1 reward for five nose pokes) schedule for 4 days, and then the experimental PR schedule $\left(\mathrm{PR}=5 \mathrm{e}^{(R \times 0.2)}-5\right.$, where $R$ equals the number of rewards earned plus one) [31, 32]. The final ratio completed, or breakpoint, was assessed at the earlier of $5 \mathrm{~min}$ without nose pokes or $45 \mathrm{~min}$. After stable baseline performance was achieved, mice were treated, in a counterbalanced fashion, with vehicle and $0.5 \mathrm{mg} / \mathrm{kg}$ LPS by a 1 week interval. Mice were tested 24 and $48 \mathrm{~h}$ after treatment.

Effort-based decision making task. To assess effort allocation a concurrent choice effort-based decision task was used $[33,34]$. Mice were restricted to $85-90 \%$ of their free feeding weight and were trained to nose poke for chocolate-flavored and grain-based Dustless Precision Pellets ( $20 \mathrm{mg}$, BioServ, Frenchtown, NJ) in operant conditioning chambers equipped with a nose-poke response unit and reward units on both the right and left walls (Med Associates, St. Albans, VT). Mice were trained on an FR1 schedule for grain-based pellets and an FR10 schedule for chocolate-flavored pellets as previously described [34]. The higher reward value of chocolate pellets was confirmed by a free feeding task where both type of pellets were freely available [34]. Training on the concurrent FR1/FR10 schedule continued until stable percent chocolate preference $(\%$ chocolate preference $=[$ chocolate pellets earned $] /[$ chocolate + grain pellets 
A

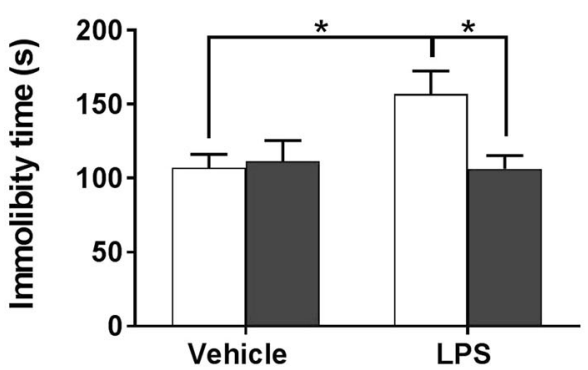

C

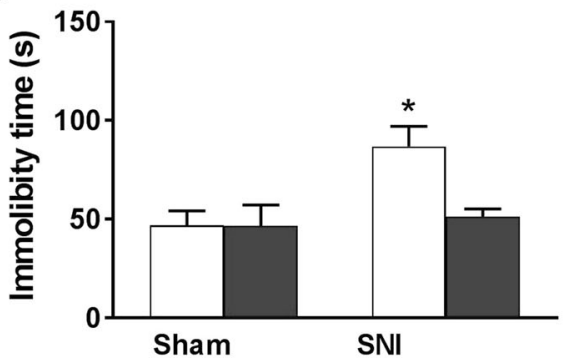

B

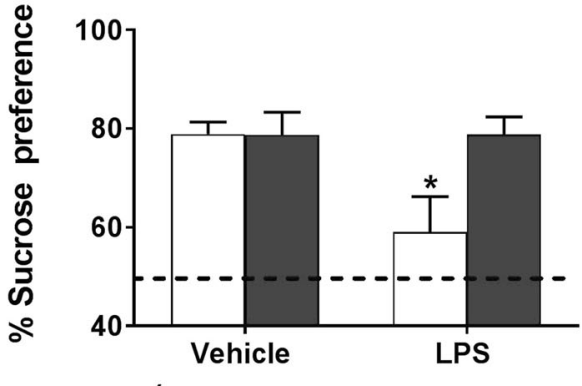

$1 d 01^{-1-}$

D

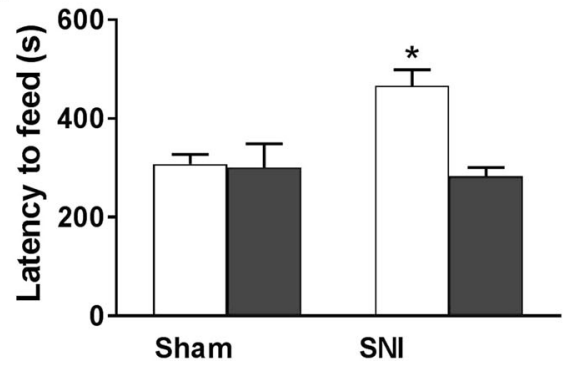

Fig. 1 Inflammation-induced depression-like behavior on pharmacological tests of antidepressants requires IDO1. Male WT mice showed enhanced immobility in the FST $24 \mathrm{~h} 0.33 \mathrm{mg} / \mathrm{kg}$ LPS (a) and suppressed sucrose preference $36-48 \mathrm{~h}$ post LPS (b), these effects were not observed in $I d o 1^{-1-}$ mice. $n=6-7$ mice/group. Further, female WT mice showed enhanced immobility in the FST 6 weeks after SNI surgery (c) and increased latency to feed in the NSF task (d), these effects were not observed in $I d o 1^{-1-}$ mice. $n=6$ mice/group. ${ }^{*} p<0.05$

earned] $\times 100$ ) was reached. Mice were treated, in a counterbalanced fashion, with vehicle and $0.33 \mathrm{mg} / \mathrm{kg}$ LPS by a 1 week interval. Mice were tested $24 \mathrm{~h}$ after treatment.

CPP. CPP was used to assess the ability of mice to associate environmental cues to a reward. SNI mice experience chronic pain, therefore, they learn to prefer a distinct environment when that environment is paired with transient pain relief provided by the analgesic retigabine [35-37]. The CPP apparatus consists of a dark and bright chamber $(18 \times 20 \mathrm{~cm})$ connected by a $15 \mathrm{~cm}$ hallway (Stoelting, Wood Dale, IL). Training was conducted as previously described [38]. Briefly, female mice started training 5 weeks after SNI surgery. On day 1 , mice freely explored the apparatus for $15 \mathrm{~min}$. On days $2-5$, mice received a morning IP injection of PBS and, after $10 \mathrm{~min}$, were placed in the dark chamber for $15 \mathrm{~min}$. In the afternoon mice received $10 \mathrm{mg} / \mathrm{kg}$ IP injection of retigabine (\#R-100, Alamone Laboratory, Jerusalem, Israel) and, after $10 \mathrm{~min}$, were placed in the bright chamber for $15 \mathrm{~min}$. On day 6, mice were again allowed to freely explore the chamber for $15 \mathrm{~min}$. Mice were video recorded on day 1 and 6 (pre- and post-conditioning) and the change in time spent in the bright compartment was quantified by EthoVision XT Software (Nodus Information Technology, Leesberg, VA).

\section{1-Methyl-DL-tryptophan (1-MT) administration}

One experiment used 1-MT (cat \#860646, Sigma-Aldrich, St. Louis, MO), a competitive inhibitor of IDO1 [39], to pharmacologically block IDO1 activity. 1-MT was prepared daily at a $4 \times$ concentration in $0.1 \mathrm{M} \mathrm{HCL}$, neutralized with $0.1 \mathrm{M} \mathrm{NaOH}$, diluted with PBS, and filtered. Mice were subcutaneously injected with $5 \mathrm{mg} 1-\mathrm{MT}$ in $0.25 \mathrm{~mL} 48 \mathrm{~h}, 24 \mathrm{~h}$, and $30 \mathrm{~min}$ prior to LPS treatment.

\section{Statistical analysis}

Statistical analyses were performed using GraphPad Prism 6.0 and Statistical Package for Social Sciences version 22. Two-way ANOVAs with between subjects genotype factor (WT vs $I d o 1^{-/-}$) and within subjects (vehicle and LPS) or between subjects (control or SNI) treatment factor were primarily used. When appropriate, repeated measures ANOVAs, $t$-tests, or one-way ANOVAs were used. Tukey post-hoc tests were conducted as necessary. All data are presented as means \pm standard error of the mean. A $p$-value of $\leq 0.05$ was considered statistically significant.

\section{RESULTS}

IDO1 mediates depression-like behavior in behavioral tests of antidepressant activity

We began by confirming the necessity of IDO1 for inflammationinduced depression-like behavior. To verify that sickness developed similarly in both genotypes and had resolved prior to testing, we examined locomotor activity at 2 and $24 \mathrm{~h}$ post LPS. As anticipated, both WT and $I d o 1^{-1-}$ mice showed a similar sickness response to $0.33 \mathrm{mg} / \mathrm{kg}$ LPS, as indicated by a $50 \%$ reduction in locomotor activity $2 \mathrm{~h}$ post injection that had resolved by $24 \mathrm{~h}$ (Figure S3). In the FST, conducted immediately after the $24 \mathrm{~h}$ locomotor assessment, LPS-treated WT mice displayed increased immobility while $I d o 1^{-1-}$ mice showed no LPS-induced increase in immobility (genotype $\times$ treatment interaction, $F(1,23)=5.14, p<$ 0.05 ) (Fig. 1a). Further, WT mice, but not $I d o 1^{-1-}$ mice, displayed a sucrose preference deficit $36-48 \mathrm{~h}$ post LPS (genotype $\times$ treatment interaction, $F(1,23)=4.71, p<0.05)$ (Fig. 1b).

Next we tested FST and NSF following SNI. We demonstrated that female SNI Ido $1^{-1-}$ mice are protected from SNI-induced FST deficits $(F(1,20)=4.33, p=0.05)$, as we previously demonstrated in male mice [12]. We demonstrate for the first time that the SNIinduced increased latency to eat in the NSF task is IDO1 dependent $(F(1,20)=7.53, p<0.05)$ (Fig. 1c, d). These changes were observed independently of changes in food consumption, locomotor activity, or body weight (Figure S4).

These sets of experiments confirm that IDO1 is critical for expression of inflammation-induced depression-like behavior in behavioral tests of antidepressants. 

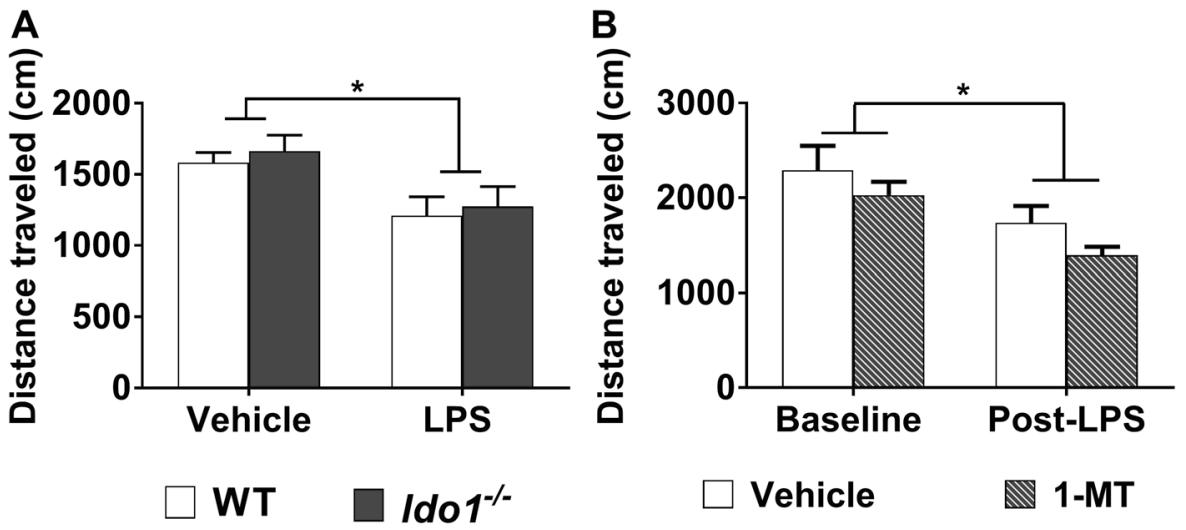

Fig. 2 LPS attenuates restricted feeding-induced anticipatory activity independently of IDO1. Male mice exposed to a restricted feeding schedule exhibit a suppression of locomotor in the $5 \mathrm{~min}$ prior to feeding $24 \mathrm{~h}$ after treatment with LPS regardless of genotype (a). This finding was confirmed using 1-MT, a competitive inhibitor of IDO1 (b). $n=6-7$ mice/group. ${ }^{*} p<0.05$

\section{Inflammation reduces reward expectancy independently of IDO1}

To evaluate inflammation-induced changes in reward expectancy, we used a food-related anticipatory activity task. In response to a restricted feeding schedule, mice display an increase in activity immediately prior to food delivery. By comparing WT free feeding and restricted mice we identified the time course for the development of this anticipatory activity (Figure S5). Next, we compared WT and $I d o 1^{-1-}$ mice all subjected to food restriction. As feeding access was ended at the time of LPS administration, there was no difference in body weight between vehicle- and LPStreated mice in either experiment (Figure S6). Continuous home cage running wheel data revealed that restricted feeding was associated with the development of anticipatory activity in both WT and $I d o 1^{-1-}$ mice (Figure S7). In response to LPS, WT, and $\mid d o 1^{-/-}$mice showed the same decrease in anticipatory activity (Fig. 2a; main effect of LPS, $F(1,21)=9.94, p<0.005)$. We confirmed these findings by blocking IDO1 with 1-MT. As with $I d o 1^{-1-}$ mice, mice treated with 1-MT showed no protection from LPS-induced reduction in anticipatory activity (Fig. 2b; main effect of LPS, $F(1,12)=29.24, p<0.001$, no group by LPS interaction). This confirms that IDO1 is not necessary for LPS-induced reductions in reward expectancy.

\section{Inflammation reduces willingness to work for reward independently of IDO1}

To evaluate the impact of inflammation on willingness to work for reward, WT and $I d o 1^{-1-}$ mice were trained on a progressive ratio schedule for food reward. In this task, the amount of work (i.e., nose pokes) required for a single chocolate pellet is rapidly increased to determine the maximum ratio for which an individual was will work (i.e., the breakpoint). There was no difference in body weight between vehicle and LPS treatment at the time of testing (Figure S8). Mice treated with LPS had a lower breakpoint at 24 and $48 \mathrm{~h}$ post treatment (significant effect of LPS, $F(1,30)=$ $5.18, p<0.05$ ) (Fig. 3). This effect was observed in both WT and $\mid d o 1^{-1-}$ mice, with no significant effect of genotype nor genotype by time or treatment interactions. This indicates that IDO1 is not necessary for LPS-induced reduction in willingness to work for reward.

\section{Inflammation shifts effort allocation independently of IDO1}

To evaluate inflammation-induced changes in effort allocation, we tested $I d o 1^{-1-}$ and WT mice in the concurrent choice task following vehicle and LPS. In this task mice could choose between nose poking on an FR1 schedule for a grain pellet or on an FR10 schedule for a chocolate pellet. LPS decreased the total number of responses in the same manner in both $I d o 1^{-/-}$and WT

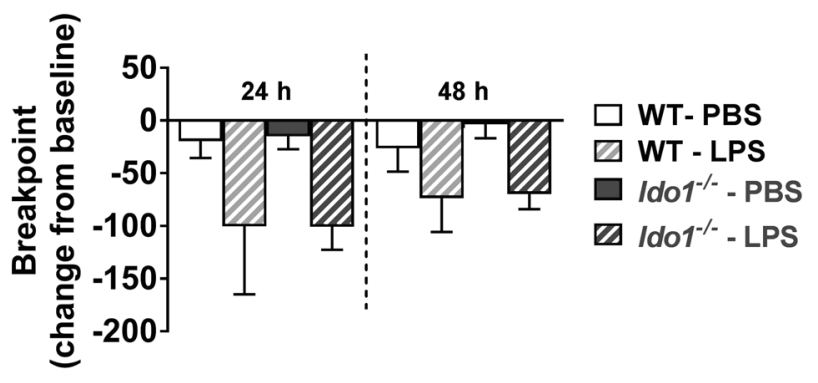

Fig. 3 LPS reduces total effort in a progressive ratio task independently of IDO1. In response to $0.5 \mathrm{mg} / \mathrm{kg}$ LPS, male mice showed a reduction in their progressive ratio breakpoint 24 and $48 \mathrm{~h}$ post LPS, $F(1,30)=5.18, p<0.05$, regardless of genotype. WT and $\mid d o 1^{-/-}$mice showed no significant differences in baseline performance. $n=8-9$ mice/group

mice $(F(1,14)=17.8, p<0.001) \quad$ (Fig. 4a).This effect was more pronounced for their willingness to work for grain $(F(1,14)=118.3$, $p<0.001)$ than their willingness to work for chocolate $(F(1,14)=$ $6.62, p<0.05$ ) despite the higher effort requirement (Fig. $4 b, c)$. Therefore, LPS resulted in a higher percent effort directed toward chocolate pellets $(F(1,14)=14.4, p<0.005)$ (Fig. $4 d)$. The effects of LPS on concurrent choice performance align with our previous observations [34]. No main effects of genotype or treatment by genotype interactions were observed.

Immediately prior to the $24 \mathrm{~h}$ post-LPS concurrent choice test, we assessed anticipatory activity by measuring locomotor activity in an open field. As would be anticipated based on the previously reward expectancy experiment, LPS treatment reduced activity prior to being given access to food rewards in the testing apparatus $(F(1,14)=10.27, p<0.01)$ (Figure S9). Neither the preference nor the latency to begin eating the chocolate pellets (Figure S10A and S10B) were significantly impacted by LPS treatment nor genotype. After concurrent choice testing, mice were returned to their home cage and provided ten chocolate and ten grain pellets to assess chocolate preference in a no effort task. All of the mice showed a continued interest in food when the effort criteria was removed, which suggests that satiation was not a performance limiting factor in the concurrent choice task (Figure S10C). Further, as the mice were at a similar body weight $24 \mathrm{~h}$ after vehicle or LPS treatment (Figure S10D), post-sickness hunger is unlikely to be a primary factor in driving the shift toward increased chocolate preference. However, further testing with a non-gustatory reward would be necessary to completely eliminate this possibility. 
A

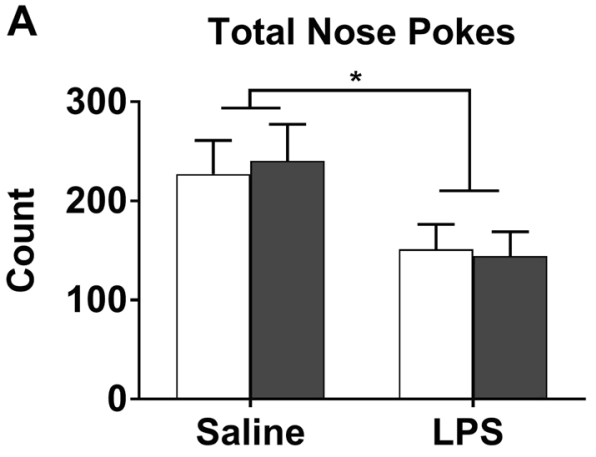

B

Nose Pokes for Grain

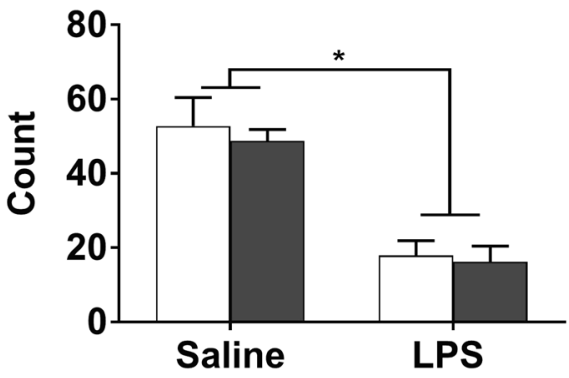

WT $\square / d o 1^{-/-}$
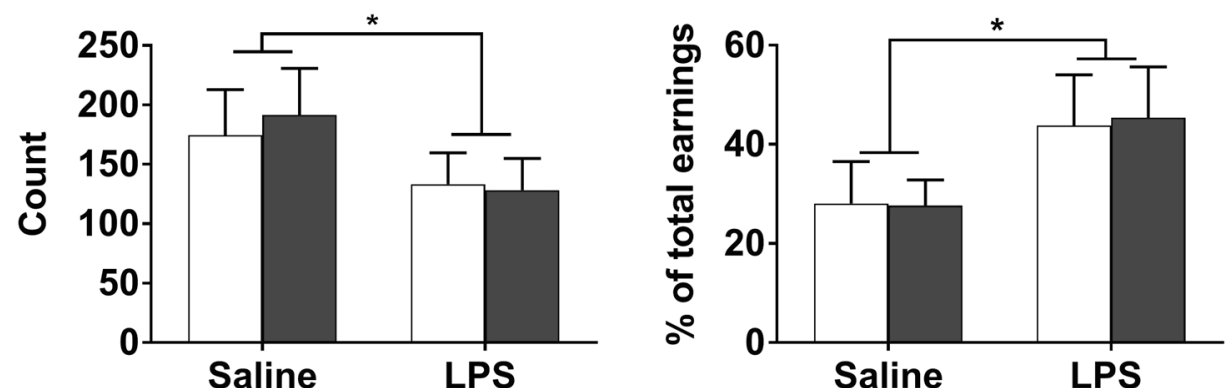

Fig. 4 LPS shift effort toward the preferred reward an effort-based decision making task independently of IDO1. In response to LPS both WT and $I d o 1^{-/-}$male mice showed a significant reduction in total nose poke responses (a). While there was reduced responding for both grain (b) and chocolate (c) pellets, the proportionally greater reduction for grain pellets lead to an increase in percent chocolate pellets earned (d). $n=8$ mice/group. ${ }^{*} p<0.05$
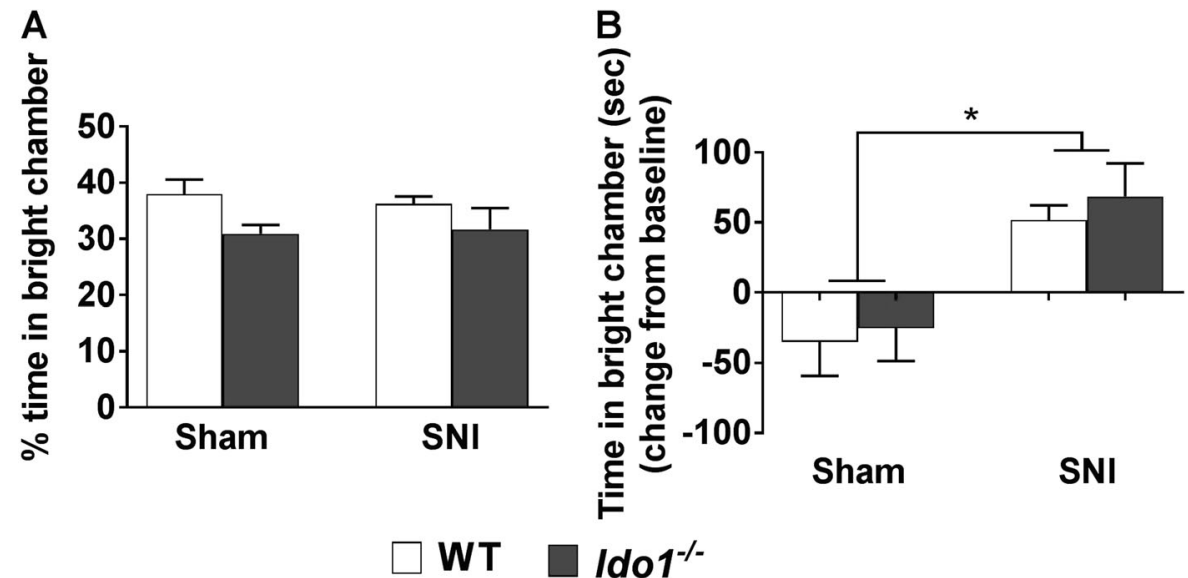

Fig. 5 SNI mice learn to associate environmental cues with the reward of pain relief independently of IDO1. Prior to conditioning (baseline) both sham and SNI female mice display a preference for the dark chamber ( $<50 \%$ time spent in the light chamber) (a). After repeated pairing of the bright chamber with analgesia and the dark chamber with vehicle, both WT and Ido ${ }^{-1-}$ SNI mice, but not Sham mice, developed a preference for the bright chamber (b). $n=6$ mice/group. ${ }^{*} p<0.05$.

In summary, the data from this experiment confirm that LPS shifts percent effort to the more highly preferred reward and that IDO1 is not necessary for this shift in effort allocation.

Inflammation-induced reward learning does not require ID01 To evaluate the contribution of IDO1 to reward learning, WT and $I d o 1^{-/-}$mice were tested in a CPP paradigm in which the development of a preference for a chamber paired with pain relief was assessed. As this test requires a model of chronic pain, it was performed on SNI mice. We verified that SNI induced evoked mechanical sensitivity (i.e., von Frey test) and spontaneous pain (i.e., weight bearing) and that pain behavior did not differ between genotypes (Figure S11). As mice generally prefer dark (Fig. 5a), the light chamber was paired with the analgesic retigabine. After four pairings of the light chamber with retigabine and the dark chamber with PBS, mice freely explored the 
apparatus in the absence of retigabine or PBS treatment. Regardless of genotype, SNI mice spent significantly more time in the light chamber (Fig. 5b), while sham treated mice showed no increased preference for the light chamber. This indicates that reward learning develops following SNI independent of IDO1.

\section{DISCUSSION}

Like other forms of depression, inflammation-induced depression is a constellation of symptoms that are likely mediated by different mechanisms. In the present experiments, we confirmed that the effects of inflammation on behavioral tests of antidepressant activity (e.g., FST and sucrose preference) are mediated by IDO1. We also demonstrated that inflammation influenced multiple components of RDoC positive valence systems including reward expectancy, willingness to work for reward, and effort allocation. Further, as $I d o 1^{-/-}$and WT mice showed no differences in any motivational behavioral task under investigation, we conclude that the effects of inflammation on alterations in positive valence systems do not dependent upon activation of the IDO1kynurenine pathway. The present experiments were conducted at a time in which IDO1 mediates performance in behavioral tests of antidepressant activity $[11-13,20]$. This indicates that inflammation-induced motivational deficits are mechanistically distinct from alterations measured by behavioral tests of antidepressant activity.

In line with the literature $[40,41]$, our data show that food-deprived mice develop anticipatory activity for a food reward. Further, they demonstrate that inflammation decreases reward expectancy. Prior work has demonstrated that inflammation decreases an individual's willingness to exert effort at the peak of inflammation-induced sickness [42, 43]. Our data demonstrate that this also occurs at a time at which sickness would have fully dissipated. Specifically, we demonstrate that the progressive ratio task is impaired for at least $48 \mathrm{~h}$ post LPS [20].

The effects observed in the anticipatory activity and progressive ratio task could be explained as a reduced sensitivity to food reward, a decrease in incentive motivation (i.e., the amount of effort an individual will engage in to obtain a reward), or increased fatigability. To determine which specific component of motivated behavior is affected, we examined the effects of LPS on performance in an effort-based decision making task that contrasts high effort/high reward and low effort/low reward modes of responding. LPS administration has already been shown to shift effort allocation toward high effort/high reward conditions in both mice and human subjects [34, 44]. In the present study, we confirmed the results in mice and showed that $I d o 1^{-1-}$ mice do not differ from WT mice in response to LPS. In contrast to our findings of a shift toward high effort/high reward, a shift from high effort/high reward to low effort/low reward has been reported in rats treated with IL-1 $\beta$ and IL-6 $[42,43]$. These differences are likely due to methodological differences in the task. Our behavioral testing was conducted $24 \mathrm{~h}$ after LPS treatment, rather than 45 or 90 min after administration of IL-6 and IL-1. Further, our task required the same mode of response (nose poking) for both forms of reward, others use free chow as the low effort/low reward option. Whatever the case, our results confirm that the effects of LPS on positively motivated behavior are not mediated by decreased sensitivity to reward or increased fatigability, but rather by a decrease in incentive motivation together with an increased sensitivity to contrast. Further, these results demonstrate that IDO1 does not mediate these effects.

Finally, the CPP paradigm allowed us to investigate motivational processes involved in the selection of a specific behavior over other possible behaviors depending on prior experience, which is central to learning theory. The motivational effect of a rewarding experience (i.e., relief from pain in chronically inflamed individuals) was assessed by pairing the less preferred compartment of a twocompartment chamber (conditioned stimuli) with the reward (unconditioned stimulus). Formation of an association between these stimuli resulted in the development of a preference for the initially less preferred compartment. These findings indicate that mice with chronic inflammatory pain learn a place preference based on relief from pain and that this learning does not dependent on IDO1.

The demonstration that IDO1 is not critical for inflammationinduced motivational changes is important in that it reveals that distinct neurobiological circuits underlie different components of inflammation-induced depression-like behavior. In terms of neurochemical mechanisms there is evidence that the dimension of depression assessed by behavioral tests of antidepressant activity is mediated by NMDA receptor activation due to IDO1induced formation of neurotoxic kynurenine metabolites [13]. However, our present findings show that this pathway does not mediate motivational components of inflammation-induced depression. Of note, the dissociation we observed in terms of IDO1 dependency between the sucrose preference test and the tests of motivated behavior used in the present experiments is likely attributable to the consummatory versus the appetitive dimension of the reward processes that is differentially involved in these tests. Further research is clearly needed to explore this possibility.

A likely candidate mechanism for the motivational effects of inflammation is represented by alterations in dopaminergic mesostriatal and mesolimbic pathways [8, 45, 46]. These pathways modulate effort-related motivational behavior assessed by the progressive ratio and concurrent choice tasks $[42,43]$. Inflammation is well known to have a profound impact on dopaminergic neurotransmission. For example, injections of LPS into the substantia nigra result in a severe loss of dopamine neurons $[47,48]$. Further, systemically administered LPS has been shown to reduce the firing rate of dopamine neurons in the ventral tegmental area [49] and increase the activity of dopamine transporters [50]. Additionally, it has been demonstrated that a local inflammatory insult (i.e., intraplantar carrageenan) can decrease dopamine release in the insular cortex [51]. The effects of inflammation on dopaminergic neurotransmission may be direct, such as through inflammation-induced oxidative damage to dopaminergic neurons $[52,53]$, or indirect, such as through inhibition of the orexinergic input to the dopaminergic neuronal network [54, 55]. Further work is clearly needed to evaluate this and other possible mechanisms of inflammation-induced motivational deficits such that interventions can be developed to address these distinct symptoms and, thereby, allow for more personalized and complete depression treatment.

\section{ACKNOWLEDGEMENTS}

This research was supported by the National Institutes of Health (R01 CA193522 and R21 MH104694 to R.D., R01 NS073939 to A.K., R.D., and C.J.H., and an MD Anderson Cancer Center Support Grant (P30 CA016672)).

\section{ADDITIONAL INFORMATION}

Supplementary Information accompanies this paper at (https://doi.org/10.1038/ s41386-018-0075-z)

Competing interests: R.D. has received honoraria from Danone Nutricia Research that is unrelated to the present study. All remaining authors declare no competing interests.

Publisher's note: Springer Nature remains neutral with regard to jurisdictional claims in published maps and institutional affiliations. 


\section{REFERENCES}

1. Dantzer R (2018). Psychiatric disorders and inflammation. In: Cavaillon JM, Singer $M$, editors. Inflammation: from molecular and cellular mechanisms to the clinic. Wiley-VCH, Weinheim Germany.

2. Kappelmann N, Lewis G, Dantzer R, Jones PB, Khandaker GM. Antidepressant activity of anti-cytokine treatment: a systematic review and meta-analysis of clinical trials of chronic inflammatory conditions. Mol Psychiatry. 2018;23:335-343.

3. Duivis HE, Vogelzangs N, Kupper N, de Jonge P, Penninx BW. Differential association of somatic and cognitive symptoms of depression and anxiety with inflammation: findings from the Netherlands Study of Depression and Anxiety (NESDA). Psychoneuroendocrinology. 2013;38:1573-1585.

4. White J, Kivimaki M, Jokela M, Batty GD. Association of inflammation with specific symptoms of depression in a general population of older people: The English Longitudinal Study of Ageing. Brain Behav Immun. 2017;61:27-30.

5. Case SM, Stewart JC. Race/ethnicity moderates the relationship between depressive symptom severity and C-reactive protein: 2005-2010 NHANES data. Brain Behav Immun. 2014;41:101-108.

6. Kennard B, Silva S, Vitiello B, Curry J, Kratochvil C, Simons A, et al. Remission and residual symptoms after short-term treatment in the Treatment of Adolescents with Depression Study (TADS). J Am Acad Child Adolesc Psychiatry. 2006;45:1404-1411.

7. Nierenberg AA, Keefe BR, Leslie VC, Alpert JE, Pava JA, Worthington JJ 3rd, et al. Residual symptoms in depressed patients who respond acutely to fluoxetine. J Clin Psychiatry. 1999;60:221-225.

8. Felger JC, Li Z, Haroon E, Woolwine BJ, Jung MY, Hu X, et al. Inflammation is associated with decreased functional connectivity within corticostriatal reward circuitry in depression. Mol Psychiatry. 2016;21:1358-1365.

9. Insel TR. The NIMH Research Domain Criteria (RDoC) Project: precision medicine for psychiatry. Am J Psychiatry. 2014;171:395-397.

10. Laumet G, Zhou W, Dantzer R, Edralin JD, Huo X, Budac DP, et al. Upregulation of neuronal kynurenine 3-monooxygenase mediates depression-like behavior in a mouse model of neuropathic pain. Brain Behav Immun. 2017;66:94-102.

11. O'Connor JC, Lawson MA, Andre C, Moreau M, Lestage J, Castanon N, et al. Lipopolysaccharide-induced depressive-like behavior is mediated by indoleamine 2,3-dioxygenase activation in mice. Mol Psychiatry. 2009;14:511-522.

12. Zhou W, Dantzer R, Budac DP, Walker AK, Mao-Ying QL, Lee AW, et al. Peripheral indoleamine 2,3-dioxygenase 1 is required for comorbid depression-like behavior but does not contribute to neuropathic pain in mice. Brain Behav Immun. 2015;46:147-153.

13. Walker AK, Budac DP, Bisulco $S$, Lee AW, Smith RA, Beenders $B$, et al. NMDA receptor blockade by ketamine abrogates lipopolysaccharide-induced depressive-like behavior in C57BL/6J mice. Neuropsychopharmacology. 2013;38: 1609-1616.

14. Raison CL, Dantzer R, Kelley KW, Lawson MA, Woolwine BJ, Vogt G, et al. CSF concentrations of brain tryptophan and kynurenines during immune stimulation with IFN-alpha: relationship to CNS immune responses and depression. Mol Psychiatry. 2010;15:393-403.

15. Steiner J, Walter M, Gos T, Guillemin GJ, Bernstein HG, Sarnyai Z, et al. Severe depression is associated with increased microglial quinolinic acid in subregions of the anterior cingulate gyrus: evidence for an immune-modulated glutamatergic neurotransmission? J Neuroinflamm. 2011;8:94.

16. Meier TB, Drevets WC, Teague TK, Wurfel BE, Mueller SC, Bodurka J, et al. Kynurenic acid is reduced in females and oral contraceptive users: Implications for depression. Brain Behav Immun. 2018;67:59-64.

17. Henry CJ, Huang Y, Wynne A, Hanke M, Himler J, Bailey MT, et al. Minocycline attenuates lipopolysaccharide (LPS)-induced neuroinflammation, sickness behavior, and anhedonia. J Neuroinflamm. 2008;5:15.

18. Martin SA, Pence BD, Greene RM, Johnson SJ, Dantzer R, Kelley KW, et al. Effects of voluntary wheel running on LPS-induced sickness behavior in aged mice. Brain Behav Immun. 2013;29:113-123.

19. Godbout JP, Moreau M, Lestage J, Chen J, Sparkman NL, O'Connor J, et al. Aging exacerbates depressive-like behavior in mice in response to activation of the peripheral innate immune system. Neuropsychopharmacology. 2008; 33:2341-2351

20. Dantzer R, O'Connor JC, Freund GG, Johnson RW, Kelley KW. From inflammation to sickness and depression: when the immune system subjugates the brain. Nat Rev Neurosci. 2008;9:46-56.

21. Laumet G, Garriga J, Chen SR, Zhang Y, Li DP, Smith TM, et al. G9a is essential for epigenetic silencing of $\mathrm{K}(+)$ channel genes in acute-to-chronic pain transition. Nat Neurosci. 2015;18:1746-1755.

22. Salazar A, Gonzalez-Rivera BL, Redus L, Parrott JM, O'Connor JC. Indoleamine 2,3dioxygenase mediates anhedonia and anxiety-like behaviors caused by peripheral lipopolysaccharide immune challenge. Horm Behav. 2012;62:202-209.
23. Bodnoff SR, Suranyi-Cadotte B, Aitken DH, Quirion R, Meaney MJ. The effects of chronic antidepressant treatment in an animal model of anxiety. Psychopharmacology. 1988;95:298-302.

24. Singhmar P, Huo X, Eijkelkamp N, Berciano SR, Baameur F, Mei FC, et al. Critical role for Epac1 in inflammatory pain controlled by GRK2-mediated phosphorylation of Epac1. Proc Natl Acad Sci USA. 2016;113:3036-3041.

25. Chaplan SR, Bach FW, Pogrel JW, Chung JM, Yaksh TL. Quantitative assessment of tactile allodynia in the rat paw. J Neurosci Methods. 1994;53:55-63.

26. Malek N, Mrugala M, Makuch W, Kolosowska N, Przewlocka B, Binkowski M, et al. A multi-target approach for pain treatment: dual inhibition of fatty acid amide hydrolase and TRPV1 in a rat model of osteoarthritis. Pain. 2015;156:890-903.

27. Tetreault $P$, Dansereau MA, Dore-Savard L, Beaudet N, Sarret P. Weight bearing evaluation in inflammatory, neuropathic and cancer chronic pain in freely moving rats. Physiol Behav. 2011;104:495-502.

28. Grossberg AJ, Zhu X, Leinninger GM, Levasseur PR, Braun TP, Myers MG Jr., et al. Inflammation-induced lethargy is mediated by suppression of orexin neuron activity. J Neurosci. 2011;31:11376-11386.

29. Mistlberger RE. Circadian food-anticipatory activity: formal models and physiological mechanisms. Neurosci Biobehav Rev. 1994;18:171-195.

30. Stephan FK. The "other" circadian system: food as a zeitgeber. J Biol Rhythms. 2002;17:284-292.

31. Grossberg AJ, Vichaya EG, Christian DL, Molkentine JM, Vermeer DW, Gross PS, et al. Tumor-associated fatigue in cancer patients develops independently of IL1 signaling. Cancer Res. 2018;78:695-705.

32. Sharma S, Hryhorczuk C, Fulton S. Progressive-ratio responding for palatable high-fat and high-sugar food in mice. J Vis Exp. 2012;63:e3754.

33. Salamone JD, Steinpreis RE, McCullough LD, Smith P, Grebel D, Mahan K. Haloperidol and nucleus accumbens dopamine depletion suppress lever pressing for food but increase free food consumption in a novel food choice procedure. Psychopharmacology. 1991;104:515-521.

34. Vichaya EG, Hunt SC, Dantzer R. Lipopolysaccharide reduces incentive motivation while boosting preference for high reward in mice. Neuropsychopharmacology. 2014;39:2884-2890.

35. King T, Vera-Portocarrero L, Gutierrez T, Vanderah TW, Dussor G, Lai J, et al. Unmasking the tonic-aversive state in neuropathic pain. Nat Neurosci. 2009;12:1364-1366.

36. Sufka KJ. Conditioned place preference paradigm: a novel approach for analgesic drug assessment against chronic pain. Pain. 1994;58:355-366.

37. Yang Q, Wu Z, Hadden JK, Odem MA, Zuo Y, Crook RJ, et al. Persistent pain after spinal cord injury is maintained by primary afferent activity. J Neurosci. 2014;34:10765-10769.

38. Krukowski K, Ma J, Golonzhka O, Laumet GO, Gutti T, van Duzer JH, et al. HDAC6 inhibition effectively reverses chemotherapy-induced peripheral neuropathy. Pain. 2017;158:1126-1137.

39. Cady SG, Sono M. 1-Methyl-DL-tryptophan, beta-(3-benzofuranyl)-DL-alanine (the oxygen analog of tryptophan), and beta-[3-benzo(b)thienyl]-DL-alanine (the sulfur analog of tryptophan) are competitive inhibitors for indoleamine 2,3dioxygenase. Arch Biochem Biophys. 1991;291:326-333.

40. Diaz-Munoz M, Vazquez-Martinez O, Aguilar-Roblero R, Escobar C. Anticipatory changes in liver metabolism and entrainment of insulin, glucagon, and corticosterone in food-restricted rats. Am J Physiol Regul Integr Comp Physiol. 2000;279:R2048-2056.

41. Sheward WJ, Maywood ES, French KL, Horn JM, Hastings MH, Seckl JR, et al. Entrainment to feeding but not to light: circadian phenotype of VPAC2 receptornull mice. J Neurosci. 2007;27:4351-4358.

42. Nunes EJ, Randall PA, Estrada A, Epling B, Hart EE, Lee CA, et al. Effort-related motivational effects of the pro-inflammatory cytokine interleukin 1-beta: studies with the concurrent fixed ratio 5/ chow feeding choice task. Psychopharmacology. 2014;231:727-736.

43. Yohn SE, Arif Y, Haley A, Tripodi G, Baqi Y, Muller CE, et al. Effort-related motivational effects of the pro-inflammatory cytokine interleukin-6: pharmacological and neurochemical characterization. Psychopharmacology. 2016;233:3575-3586.

44. Lasselin J, Treadway MT, Lacourt TE, Soop A, Olsson MJ, Karshikoff B, et al. Lipopolysaccharide alters motivated behavior in a monetary reward task: a randomized trial. Neuropsychopharmacology. 2017;42:801-810.

45. Harrison NA, Cercignani M, Voon V, Critchley HD. Effects of inflammation on hippocampus and substantia nigra responses to novelty in healthy human participants. Neuropsychopharmacology. 2015:40:831-838.

46. Capuron L, Pagnoni G, Drake DF, Woolwine BJ, Spivey JR, Crowe RJ, et al. Dopaminergic mechanisms of reduced basal ganglia responses to hedonic reward during interferon alfa administration. Arch Gen Psychiatry. 2012;69:1044-1053.

47. Herrera AJ, Castano A, Venero JL, Cano J, Machado A. The single intranigral injection of LPS as a new model for studying the selective effects of inflammatory reactions on dopaminergic system. Neurobiol Dis. 2000;7:429-447. 
48. Castano A, Herrera AJ, Cano J, Machado A. The degenerative effect of a single intranigral injection of LPS on the dopaminergic system is prevented by dexamethasone, and not mimicked by rh-TNF-alpha, IL-1beta and IFN-gamma. J Neurochem. 2002;81:150-157.

49. Blednov YA, Benavidez JM, Geil C, Perra S, Morikawa H, Harris RA. Activation of inflammatory signaling by lipopolysaccharide produces a prolonged increase of voluntary alcohol intake in mice. Brain Behav Immun. 2011;25:Suppl 1S92-S105.

50. van Heesch F, Prins J, Konsman JP, Korte-Bouws GA, Westphal KG, Rybka J, et al. Lipopolysaccharide increases degradation of central monoamines: an in vivo microdialysis study in the nucleus accumbens and medial prefrontal cortex of mice. Eur J Pharmacol. 2014;725:55-63.

51. Coffeen U, Ortega-Legaspi JM, de Gortari P, Simon-Arceo K, Jaimes O, Amaya MI, et al. Inflammatory nociception diminishes dopamine release and increases dopamine D2 receptor mRNA in the rat's insular cortex. Mol Pain. 2010; 6:75.

52. Chung ES, Chung YC, Bok E, Baik HH, Park ES, Park JY, et al Fluoxetine prevents LPS-induced degeneration of nigral dopaminergic neurons by inhibiting microglia-mediated oxidative stress. Brain Res 2010;1363:143-150.

53. Qin L, Liu Y, Hong JS, Crews FT. NADPH oxidase and aging drive microglial activation, oxidative stress, and dopaminergic neurodegeneration following systemic LPS administration. Glia. 2013;61:855-868.

54. Korotkova TM, Sergeeva OA, Eriksson KS, Haas HL, Brown RE. Excitation of ventral tegmental area dopaminergic and nondopaminergic neurons by orexins/ hypocretins. J Neurosci. 2003;23:7-11.

55. Calipari ES, Espana RA. Hypocretin/orexin regulation of dopamine signaling: implications for reward and reinforcement mechanisms. Front Behav Neurosci. 2012;6:54.

56. Center for Behavioral Health Statistics and Quality. (2015). Behavioral health trends in the United States: Results from the 2014 National Survey on Drug Use and Health (HHS Publication No. SMA 15-4927, NSDUH Series H-50). Retrieved from https://www.samhsa.gov/data/

57. Yalcin I, Bohren Y, Waltisperger E, Sage-Ciocca D, Yin JC, Freund-Mercier MJ, et al. A time-dependent history of mood disorders in a murine model of neuropathic pain. Biol Psychiatry. 2011;70:946-953. 